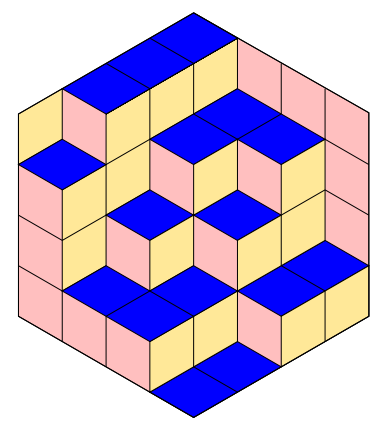

ALGEBRAIC COMBINATORICS

Trevor Hyde

Liminal reciprocity and factorization statistics

Volume 2, issue 4 (2019), p. 521-539.

<http://alco.centre-mersenne.org/item/ALCO_2019__2_4_521_0>

(c) The journal and the authors, 2019.

Some rights reserved.

(c) BY This article is licensed under the

Creative Commons ATtribution 4.0 International LiCEnSE.

http://creativecommons.org/licenses/by/4.0/

Access to articles published by the journal Algebraic Combinatorics on the website http://alco.centre-mersenne.org/ implies agreement with the Terms of Use (http://alco.centre-mersenne.org/legal/).

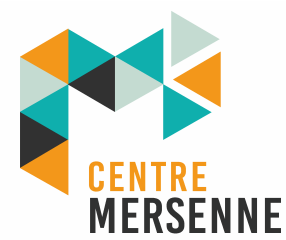

Algebraic Combinatorics is member of the Centre Mersenne for Open Scientific Publishing www.centre-mersenne.org 


\title{
Liminal reciprocity and factorization statistics
}

\author{
Trevor Hyde
}

\begin{abstract}
Let $M_{d, n}(q)$ denote the number of monic irreducible polynomials in $\mathbb{F}_{q}\left[x_{1}, x_{2}, \ldots, x_{n}\right]$ of degree $d$. We show that for a fixed degree $d$, the sequence $M_{d, n}(q)$ converges coefficientwise to an explicitly determined rational function $M_{d, \infty}(q)$. The limit $M_{d, \infty}(q)$ is related to the classic necklace polynomial $M_{d, 1}(q)$ by an involutive functional equation we call liminal reciprocity. The limiting first moments of factorization statistics for squarefree polynomials are expressed in terms of symmetric group characters as a consequence of liminal reciprocity, giving a liminal analog of a result of Church, Ellenberg, and Farb.
\end{abstract}

\section{INTRODUCTION}

Let $\mathbb{F}_{q}$ be a field with $q$ elements. How many degree $d$ polynomials in $\mathbb{F}_{q}\left[x_{1}, x_{2}, \ldots, x_{n}\right]$ are irreducible? Let $M_{d, n}(q)$ denote the number of irreducible monic ${ }^{(1)}$ polynomials in $\mathbb{F}_{q}\left[x_{1}, x_{2}, \ldots, x_{n}\right]$ of total degree $d$. When $n=1, M_{d, 1}(q)$ is given by the $d$ th necklace polynomial

$$
M_{d, 1}(q):=\frac{1}{d} \sum_{e \mid d} \mu(e) q^{d / e},
$$

where $\mu$ is the Möbius function. There does not appear to be a simple formula for $M_{d, n}(q)$ analogous to (1) when $n>1$. In Lemma $2.1 M_{d, n}(q)$ is shown to be a recursively computable polynomial in $q$ for all $n \geqslant 1$. The table below gives the low degree terms of $M_{3, n}(q)$ for small $n$.

\begin{tabular}{|l|l|}
\hline$n$ & $M_{3, n}(q)$ \\
\hline 1 & $-\frac{1}{3} q+\frac{1}{3} q^{3}$ \\
\hline 2 & $-\frac{1}{3} q-\frac{1}{3} q^{2}+\frac{1}{3} q^{3}-q^{5}-\frac{2}{3} q^{6}+\ldots$ \\
\hline 3 & $-\frac{1}{3} q-\frac{1}{3} q^{2}+q^{4}+q^{5}+\frac{1}{3} q^{6}-q^{7}+\ldots$ \\
\hline 4 & $-\frac{1}{3} q-\frac{1}{3} q^{2}+\frac{2}{3} q^{4}+2 q^{5}+\frac{7}{3} q^{6}+2 q^{7}+\ldots$ \\
\hline 5 & $-\frac{1}{3} q-\frac{1}{3} q^{2}+\frac{2}{3} q^{4}+\frac{5}{3} q^{5}+\frac{10}{3} q^{6}+4 q^{7}+\ldots$ \\
\hline 6 & $-\frac{1}{3} q-\frac{1}{3} q^{2}+\frac{2}{3} q^{4}+\frac{5}{3} q^{5}+3 q^{6}+5 q^{7}+\ldots$ \\
\hline 7 & $-\frac{1}{3} q-\frac{1}{3} q^{2}+\frac{2}{3} q^{4}+\frac{5}{3} q^{5}+3 q^{6}+\frac{14}{3} q^{7}+\ldots$ \\
\hline
\end{tabular}

Manuscript received 27th April 2018, revised 20th June 2018 and 11th July 2018, accepted 11th July 2018.

KEYWORDS. necklace polynomial, finite fields, reciprocity.

ACKNOWLEDGEMENTS. The author was partially supported by DMS-1701576.

${ }^{(1)}$ By monic in a multivariate polynomial ring we mean an $\mathbb{F}_{q}^{\times}$-orbit of polynomials under scaling. 
The table suggests that the sequence of polynomials $M_{3, n}(q)$ converges coefficientwise as the number of variables $n$ increases. We prove this to be the case for all $d \geqslant 1$.

THEOREM 1.1. Let $M_{d, n}(q)$ be the number of irreducible degree d monic polynomials in $\mathbb{F}_{q}\left[x_{1}, x_{2}, \ldots, x_{n}\right]$. Then $M_{d, n}(q)$ is a polynomial in $q$ and for each $d \geqslant 1$ the sequence of polynomials $M_{d, n}(q)$ converges coefficientwise (that is, with respect to the q-adic topology) in the formal power series ring $\mathbb{Q} \llbracket q \rrbracket$ to the rational function

$$
M_{d, \infty}(q):=-\frac{1}{d} \sum_{e \mid d} \mu(e)\left(\frac{1}{1-\frac{1}{q}}\right)^{d / e} .
$$

In particular $M_{d, \infty}(q)$ satisfies the functional equation,

$$
M_{d, \infty}(q)=-M_{d, 1}\left(\frac{1}{1-\frac{1}{q}}\right) .
$$

Furthermore the rate of convergence of $M_{d, n}(q)$ is bounded by the congruence

$$
M_{d, n}(q) \equiv M_{d, \infty}(q) \bmod q^{n+1} .
$$

The fractional linear transformation $q \mapsto \frac{1}{1-\frac{1}{q}}$ is an involution, hence (2) is equivalent to

$$
M_{d, 1}(q)=-M_{d, \infty}\left(\frac{1}{1-\frac{1}{q}}\right) .
$$

This functional equation relating irreducible polynomial counts in one and infinitely many variables is the first instance of a phenomenon we call liminal reciprocity.

1.1. Liminal Reciprocity For type polynomials. Let Poly ${ }_{d, n}\left(\mathbb{F}_{q}\right)$ denote the set of monic polynomials in $\mathbb{F}_{q}\left[x_{1}, x_{2}, \ldots, x_{n}\right]$ of total degree $d$. Since the polynomial ring $\mathbb{F}_{q}\left[x_{1}, x_{2}, \ldots, x_{n}\right]$ has unique factorization, each $f \in \operatorname{Poly}_{d, n}\left(\mathbb{F}_{q}\right)$ has a well-defined factorization type. The factorization type of a polynomial $f \in \operatorname{Poly}_{d, n}\left(\mathbb{F}_{q}\right)$ is the partition $\lambda \vdash d$ given by the degrees of the $\mathbb{F}_{q}$-irreducible factors of $f$.

REMARK 1.2. The factorization type of a polynomial does not record the multiplicities of factors, only the degrees of the irreducible factors. For example, the polynomials $x^{2}$ and $x(x+1)$ both have factorization type $\left(1^{2}\right)$ since they each have two linear factors.

DeFinition 1.3. If $\lambda \vdash d$ is a partition, then the $\lambda$-type polynomial $T_{\lambda, n}(q)$ is the number of elements in Poly $_{d, n}\left(\mathbb{F}_{q}\right)$ with factorization type $\lambda$. Similarly the squarefree $\lambda$-type polynomial $T_{\lambda, n}^{\mathrm{sf}}(q)$ is the number of squarefree elements in $\operatorname{Poly}_{d, n}\left(\mathbb{F}_{q}\right)$ with factorization type $\lambda$. The type polynomials may be expressed in terms of $M_{d, n}(q)$ as

$$
T_{\lambda, n}(q):=\prod_{j \geqslant 1}\left(\left(\begin{array}{c}
M_{j, n}(q) \\
m_{j}(\lambda)
\end{array}\right)\right) \quad T_{\lambda, n}^{\mathrm{sf}}(q):=\prod_{j \geqslant 1}\left(\begin{array}{c}
M_{j, n}(q) \\
m_{j}(\lambda)
\end{array}\right),
$$

where $m_{j}(\lambda)$ is the number of parts of $\lambda$ of size $j,\left(\begin{array}{c}x \\ m\end{array}\right):=\frac{1}{m !} x(x-1) \cdots(x-m+1)$ is the usual binomial coefficient, and $\left(\left(\begin{array}{c}x \\ m\end{array}\right)\right):=\frac{1}{m !} x(x+1) \cdots(x+m-1)$. Recall that $\left(\begin{array}{l}x \\ m\end{array}\right)$ counts the number of subsets of size $m$ in a set of size $x$ and $\left(\left(\begin{array}{c}x \\ m\end{array}\right)\right)$ counts the number of subsets of size $m$ with repetition in a set of size $x$.

It follows from Theorem 1.1 that the coefficientwise limits

$$
T_{\lambda, \infty}(q):=\lim _{n \rightarrow \infty} T_{\lambda, n}(q) \quad T_{\lambda, \infty}^{\mathrm{sf}}(q):=\lim _{n \rightarrow \infty} T_{\lambda, n}^{\mathrm{sf}}(q)
$$

converge to rational functions. Our next result is a version of liminial reciprocity for type polynomials. 
THEOREM 1.4 (Liminal reciprocity). Let $\lambda$ be a partition and let $\ell(\lambda):=\sum_{j \geqslant 1} m_{j}(\lambda)$ be the number of parts of $\lambda$. Then the following identities hold in $\mathbb{Q}(q)$,

$$
\begin{aligned}
& T_{\lambda, \infty}(q)=(-1)^{\ell(\lambda)} T_{\lambda, 1}^{\mathrm{sf}}\left(\frac{1}{1-\frac{1}{q}}\right) \\
& T_{\lambda, \infty}^{\mathrm{sf}}(q)=(-1)^{\ell(\lambda)} T_{\lambda, 1}\left(\frac{1}{1-\frac{1}{q}}\right)
\end{aligned}
$$

These identities are involutive in the sense that interchanging the $\infty$ and 1 subscripts gives an equivalent statements. The new feature appearing in Theorem 1.4 is the relationship between squarefree polynomials and general polynomials of a given factorization type. This connection is closely related to Stanley's combinatorial reciprocity phenomenon [15] (see Section 1.3.)

1.2. Liminal First moments of SQUAREFree FaCtorization Statistics. A function $P$ defined on $\operatorname{Poly}_{d, n}\left(\mathbb{F}_{q}\right)$ is called a factorization statistic if $P(f)$ depends only on the factorization type of $f$. In [10] we expressed the moments of factorization statistics on the set of univariate polynomials $(n=1)$ in terms of the symmetric group representations carried by the cohomology of point configurations in Euclidean space. (See Section 3 for definitions.) Note that $\operatorname{Poly}_{d, n}^{\mathrm{sf}}\left(\mathbb{F}_{q}\right)$ denotes the subset of squarefree polynomials in $\operatorname{Poly}_{d, n}\left(\mathbb{F}_{q}\right)$.

Theorem 1.5 ([10, Thm. 2.2, Thm. 2.3]). Let $P$ be a factorization statistic, and let $\psi_{d}^{k}, \phi_{d}^{k}$ be the characters of the $S_{d}$-representations $H^{2 k}\left(\operatorname{PConf}_{d}\left(\mathbb{R}^{3}\right), \mathbb{Q}\right)$ and $H^{k}\left(\mathrm{PConf}_{d}\left(\mathbb{R}^{2}\right), \mathbb{Q}\right)$ respectively. Then

$$
\begin{aligned}
\sum_{f \in \operatorname{Poly}_{d, 1}\left(\mathbb{F}_{q}\right)} P(f) & =\sum_{k=0}^{d-1}\left\langle P, \psi_{d}^{k}\right\rangle q^{d-k} \\
\sum_{f \in \operatorname{Poly}_{d, 1}^{\text {sf }}\left(\mathbb{F}_{q}\right)} P(f) & =\sum_{k=0}^{d-1}(-1)^{k}\left\langle P, \phi_{d}^{k}\right\rangle q^{d-k},
\end{aligned}
$$

where $\langle P, Q\rangle=\frac{1}{d !} \sum_{\tau \in S_{d}} P(\tau) Q(\tau)$ is the standard inner product of class functions on $S_{d}$.

The squarefree case (2) of Theorem 1.5 is due to Church, Ellenberg, and Farb [6, Prop. 4.1]. The general polynomial case (1) was shown by the author [10] using different methods which also led to a new proof of the squarefree case. Theorem 1.5 provides a bridge between the arithmetic and combinatorics of factorization statistics on one hand and the geometry and representation theory of configuration spaces on the other.

Numerical experiments suggest there are not direct analogs of Theorem 1.5 for polynomials in $n>1$ variables. However, an analog does emerge in the liminal squarefree case.

THEOREM 1.6. Let $P$ be a factorization statistic, and let $\sigma_{d}^{k}$ be the character of the $S_{d}$-representation

$$
\Sigma_{d}^{k}:=\bigoplus_{j=k}^{d-1} \operatorname{sgn}_{d} \otimes H^{2 j}\left(\operatorname{PConf}_{d}\left(\mathbb{R}^{3}\right), \mathbb{Q}\right)^{\oplus\left(\begin{array}{l}
j \\
k
\end{array}\right) .}
$$


For each $n$, the first moment $\sum_{f \in \operatorname{Poly}_{d, n}^{\mathrm{sf}}\left(\mathbb{F}_{q}\right)} P(f)$ is a polynomial in $q$ and

$$
\lim _{n \rightarrow \infty} \sum_{f \in \operatorname{Poly}_{d, n}^{\mathrm{sf}}\left(\mathbb{F}_{q}\right)} P(f)=\frac{1}{(1-q)^{d}} \sum_{k=0}^{d-1}(-1)^{k}\left\langle P, \sigma_{d}^{k}\right\rangle q^{d-k},
$$

where the limit is taken coefficientwise in $\mathbb{Q} \llbracket q \rrbracket$.

REMARK 1.7. By considering arbitrary factorization statistics $P$ our results also determine higher moments of $P$, as the $k$ th moment of $P$ is the first moment of $P^{k}$.

Since the limit in Theorem 1.6 is taken coefficientwise, the representation theoretic interpretation of first moments manifests for sufficiently large $n$. For example, let $L$ be the linear factor statistic where $L(f)$ is the number of linear factors of $f$. The following table shows the first moment of $L$ on $\operatorname{Poly}_{3, n}^{\mathrm{sf}}\left(\mathbb{F}_{q}\right)$ scaled by $(1-q)^{3}$.

\begin{tabular}{|l|l|}
\hline$n$ & $(1-q)^{3} \sum_{f \in \operatorname{Poly}_{3, n}^{\mathrm{sf}}\left(\mathbb{F}_{q}\right)} L(f)$ \\
\hline 1 & $q-5 q^{2}+10 q^{3}-10 q^{4}+5 q^{5}-q^{6}$ \\
\hline 2 & $q-4 q^{2}+2 q^{3}+7 q^{4}-6 q^{5}-3 q^{6}+2 q^{7}+q^{8}+q^{9}-q^{10}$ \\
\hline 3 & $q-4 q^{2}+3 q^{3}-q^{4}+7 q^{5}-6 q^{6}-3 q^{8}+3 q^{9}-q^{11}+q^{12}+q^{14}-q^{15}$ \\
\hline 4 & $q-4 q^{2}+3 q^{3}-q^{5}+7 q^{6}-6 q^{7}-3 q^{10}+3 q^{11}-q^{16}+q^{17}+q^{20}-q^{21}$ \\
\hline 5 & $q-4 q^{2}+3 q^{3}-q^{6}+7 q^{7}-6 q^{8}-3 q^{12}+3 q^{13}-q^{22}+q^{23}+q^{27}-q^{28}$ \\
\hline
\end{tabular}

From this table and the convergence bound in Theorem 1.1 we conclude that

$$
\sum_{f \in \text { Poly }_{3, n}^{\text {sf }}\left(\mathbb{F}_{q}\right)} L(f)=\frac{q-4 q^{2}+3 q^{3}+O\left(q^{n+1}\right)}{(1-q)^{3}} .
$$

It then follows from Theorem 1.6 that

$$
\left\langle L, \sigma_{3}^{2}\right\rangle=1 \quad\left\langle L, \sigma_{3}^{1}\right\rangle=4 \quad\left\langle L, \sigma_{3}^{0}\right\rangle=3 .
$$

Note that these inner products are positive integers: this reflects that $L$, viewed as a class function of the symmetric group, is the character of the standard permutation representation.

REMARK 1.8. The table above also illustrates a higher stability in the coefficients. For example, the coefficient of $q^{n+2}$ is 7 in the numerator of the first moment of $L$ for all $n \geqslant 2$. Since these exponents grow with $n$, these terms vanish in the limit as $n \rightarrow \infty$. This higher stability persists more generally; it could be an interesting direction for future work.

Liminal reciprocity gives a new method to compute the expected values of factorization statistics for univariate polynomials. As an example we compute the expected value of the sign function $\operatorname{sgn}_{d}$, where $\operatorname{sgn}_{d}(\lambda)=(-1)^{d-\ell(\lambda)}$.

Proposition 1.9. Let $d \geqslant 1$.

(1) The expected value $E_{d, 1}\left(\operatorname{sgn}_{d}\right)$ of the sign statistic on the set $\operatorname{Poly}_{d, 1}\left(\mathbb{F}_{q}\right)$ is given by

$$
E_{d, 1}\left(\operatorname{sgn}_{d}\right):=\frac{1}{q^{d}} \sum_{f \in \operatorname{Poly}_{d, 1}\left(\mathbb{F}_{q}\right)} \operatorname{sgn}_{d}(f)=\frac{1}{q^{\lfloor d / 2\rfloor}} .
$$


(2) The limiting expected value $E_{d, \infty}^{\mathrm{sf}}\left(\operatorname{sgn}_{d}\right)$ of the sign statistic on the set Poly $_{d, n}^{\mathrm{sf}}\left(\mathbb{F}_{q}\right)$ as $n \rightarrow \infty$ is given by

$$
E_{d, \infty}^{\mathrm{sf}}\left(\operatorname{sgn}_{d}\right):=\lim _{n \rightarrow \infty} \frac{1}{P_{d, n}^{\mathrm{sf}}(q)} \sum_{f \in \operatorname{Poly}_{d, n}^{\mathrm{sf}}\left(\mathbb{F}_{q}\right)} \operatorname{sgn}_{d}(f)=\left(\frac{1}{1-\frac{1}{q}}\right)^{\lfloor d / 2\rfloor},
$$

where the limit is taken $1 / q$-adically.

Proposition 1.9 is equivalent to a result of Carlitz arrived at by other means. See Proposition 3.4 and the discussion that follows.

1.3. Related WORK. Carlitz $[4,5]$ studied the asymptotic behavior of $M_{d, n}(q)$ for $n \geqslant 1$. In the language of this paper his main result is as follows.

TheOREM 1.10 ([4, Sec. 3.]). For $d, n \geqslant 1$, let $m_{d, n}:=\operatorname{deg} M_{d, n}(q)$. Then $m_{d, n}=$ $\left(\begin{array}{c}d+n \\ d\end{array}\right)-1$ and the sequence $M_{d, n}(q) / q^{m_{d, n}}$ of polynomials in $1 / q$ converges coefficientwise in $\mathbb{Q} \llbracket \frac{1}{q} \rrbracket$ to

$$
\lim _{n \rightarrow \infty} \frac{M_{d, n}(q)}{q^{m_{d, n}}}=\frac{1}{1-\frac{1}{q}} .
$$

This work was subsequently refined and extended in $[1,7,8,16,17]$. Our Theorem 1.1 may be interpreted as a determination of the $q$-adic asymptotics of $M_{d, n}(q)$ as $n \rightarrow \infty$. In other words Carlitz studied the limiting behavior of the leading terms of $M_{d, n}(q)$ and we study the limiting behavior of the low degree terms.

The liminal reciprocity identities (Theorem 1.1 and Theorem 1.4) were discovered empirically. We would be interested to know if there is a geometric or combinatorial interpretation of these results. The proof of liminal reciprocity for type polynomials (Theorem 1.4) passes through a well-known example of Stanley's combinatorial reciprocity phenomenon [15, Ex. 1.1]. Combinatorial reciprocity is a family of dualities between related combinatorial problems which concretely takes the form of functional equations similar to our liminal reciprocity identities. However, the precise relationship between liminal and combinatorial reciprocity remains unclear. Finding more examples of liminal reciprocity may shed some light on this phenomenon.

The relationship between the liminal first moments of squarefree factorization statistics and representations of the symmetric group parallels our results in [10]. Church, Ellenberg, and Farb [6] connected first moments of squarefree factorization statistics for univariate polynomials and the cohomology of point configurations in $\mathbb{R}^{2}$ with their twisted Grothendieck-Lefschetz formula for squarefree polynomials. They deduce the asymptotic stability of first moments (as $d \rightarrow \infty$ ) as a consequence of representation stability. We extend this connection to general univariate polynomials in $[10$, Thm. 2.7]. However, this connection does not extend to liminal first moments; the representations $\Sigma_{d}^{k}$ does not exhibit representation stability.

The results in [10] are expressed in terms of expected values of factorization statistics. In this paper we focus on first moments as they lead to a cleaner statement for Theorem 1.6. The only difference between expected values and first moments of factorization statistics is whether or not one divides by the "total mass" of the space of polynomials considered. This difference is simply a factor of $q^{d}$ for general univariate polynomials, but is more subtle for squarefree polynomials and multivariate polynomials as it affects the family of characters determining the coefficients. The equivalence between Theorem 1.5 (2) and [10, Thm. 2.3] follows from [11, Prop. 4.2]. Alternatively, Theorem 1.5 (2) appears as stated in [6, Prop. 4.1].

In a subsequent paper [9] we study the vanishing of the polynomials $M_{d, n}(q)$ at roots of unity and the relation of $M_{d, n}(q)$ to geometry. For a field $K$ let $\operatorname{Irr}_{d, n}(K)$ 
denote the collection of all $K$-irreducible monic polynomials of total degree $d$ in $K\left[x_{1}, x_{2}, \ldots, x_{n}\right]$. If $K=\mathbb{R}$ or $\mathbb{C}$, then $\operatorname{Irr}_{d, n}(K)$ inherits a subspace topology from the projective space of all non-zero monic polynomials of degree at most $d$. We show that the values of $M_{d, n}(q)$ at $q= \pm 1$ compute the compactly supported Euler characteristics of these spaces.

THEOREM $1.11([9])$. Let $d, n \geqslant 1$ and let $\chi_{c}$ be the compactly supported Euler characteristic, then

$$
\begin{aligned}
& \chi_{c}\left(\operatorname{Irr}_{d, n}(\mathbb{C})\right)=M_{d, n}(1)= \begin{cases}n & \text { if } d=1 \\
0 & \text { otherwise. }\end{cases} \\
& \chi_{c}\left(\operatorname{Irr}_{d, n}(\mathbb{R})\right)=M_{d, n}(-1)= \begin{cases}b_{k} & \text { if } d=2^{k} \\
0 & \text { otherwise. }\end{cases}
\end{aligned}
$$

where $n=\sum_{k \geqslant 0} b_{k} 2^{k}$ is the unique expression of $n$ as an alternating sum of an even number of powers of 2 .

\section{Polynomial factorization statistics}

Let $\mathbb{F}_{q}$ be a finite field. Recall that we define a monic polynomial in $\mathbb{F}_{q}\left[x_{1}, x_{2}, \ldots, x_{n}\right]$ to be an $\mathbb{F}_{q}^{\times}$-orbit of polynomials under scaling. Let Poly ${ }_{d, n}\left(\mathbb{F}_{q}\right)$ be the set of all total degree $d$ monic polynomials in $\mathbb{F}_{q}\left[x_{1}, x_{2}, \ldots, x_{n}\right]$. For each $m \geqslant 1$ let $\operatorname{Poly}_{d, n}^{m}\left(\mathbb{F}_{q}\right) \subseteq$ Poly $_{d, n}\left(\mathbb{F}_{q}\right)$ be the subset of those polynomials with all factors of multiplicity at most $m$. There is a filtration

$$
\operatorname{Poly}_{d, n}^{\mathrm{sf}}\left(\mathbb{F}_{q}\right):=\operatorname{Poly}_{d, n}^{1}\left(\mathbb{F}_{q}\right) \subseteq \operatorname{Poly}_{d, n}^{2}\left(\mathbb{F}_{q}\right) \subseteq \operatorname{Poly}_{d, n}^{3}\left(\mathbb{F}_{q}\right) \subseteq \ldots \subseteq \operatorname{Poly}_{d, n}\left(\mathbb{F}_{q}\right),
$$

where $\operatorname{Poly}_{d, n}^{\mathrm{sf}}\left(\mathbb{F}_{q}\right)$ is the set of the squarefree polynomials.

Recall that $\mathbb{F}_{q}\left[x_{1}, x_{2}, \ldots, x_{n}\right]$ is a unique factorization domain, hence every element of $\operatorname{Poly}_{d, n}\left(\mathbb{F}_{q}\right)$ has a unique factorization as a product of irreducible monic polynomials. The factorization type of $f \in \operatorname{Poly}_{d, n}\left(\mathbb{F}_{q}\right)$ is the partition of $d$ given by the degrees of the $\mathbb{F}_{q}$-irreducible factors of $f$. If $\lambda$ is a partition of $d$, then let $\operatorname{Poly}_{\lambda, n}\left(\mathbb{F}_{q}\right)$ denote the set of all $f \in \operatorname{Poly}_{d, n}\left(\mathbb{F}_{q}\right)$ with factorization type $\lambda$. For $m \geqslant 1$, let $\operatorname{Poly}_{\lambda, n}^{m}\left(\mathbb{F}_{q}\right):=\operatorname{Poly}_{d, n}^{m}\left(\mathbb{F}_{q}\right) \cap \operatorname{Poly}_{\lambda, n}\left(\mathbb{F}_{q}\right)$. If $\lambda=(d)$ is the partition with one part, let $\operatorname{Irr}_{d, n}\left(\mathbb{F}_{q}\right):=\operatorname{Poly}_{(d), n}\left(\mathbb{F}_{q}\right)$ be the set of monic, irreducible, total degree $d$ polynomials.

Lemma 2.1 shows that the cardinality of each of the sets just defined is given by a polynomial in the size of the field $q$.

LEMma 2.1. For any $d, n \geqslant 1$,

(1) $\left|\operatorname{Poly}_{d, n}\left(\mathbb{F}_{q}\right)\right|=P_{d, n}(q)$, where

$$
P_{d, n}(q):=\frac{q^{\left(\begin{array}{c}
d+n \\
n
\end{array}\right)}-q^{\left(\begin{array}{c}
d+n-1 \\
n
\end{array}\right)}}{q-1}=q^{\left(\begin{array}{c}
d+n-1 \\
n
\end{array}\right)} \frac{q^{\left(\begin{array}{c}
d+n-1 \\
n-1
\end{array}\right)}-1}{q-1} .
$$

(2) $M_{d, n}(q):=\left|\operatorname{Irr}_{d, n}\left(\mathbb{F}_{q}\right)\right|$ is a polynomial in $q$ with rational coefficients.

(3) For every partition $\lambda \vdash d$,

$$
\begin{aligned}
& \left|\operatorname{Poly}_{\lambda, n}\left(\mathbb{F}_{q}\right)\right|=T_{\lambda, n}(q):=\prod_{j \geqslant 1}\left(\left(\begin{array}{c}
M_{j, n}(q) \\
m_{j}(\lambda)
\end{array}\right)\right), \\
& \left|\operatorname{Poly}_{\lambda, n}^{\mathrm{sf}}\left(\mathbb{F}_{q}\right)\right|=T_{\lambda, n}^{\mathrm{sf}}(q):=\prod_{j \geqslant 1}\left(\begin{array}{c}
M_{j, n}(q) \\
m_{j}(\lambda)
\end{array}\right) .
\end{aligned}
$$

where $\left(\left(\begin{array}{c}x \\ m\end{array}\right)\right):=\left(\begin{array}{c}x+m-1 \\ m\end{array}\right)$ is the number of subsets with repetition of size $m$ chosen from an $x$ element set. 
Proof. (1) There are $q^{\left(\begin{array}{c}d+n \\ n\end{array}\right)}$ polynomials in $n$ variables of degree at most $d$. Hence there are $q^{\left(\begin{array}{c}d+n \\ n\end{array}\right)}-q^{\left(\begin{array}{c}d+n-1 \\ n\end{array}\right)}$ polynomials in $n$ variables of degree exactly $d$. Taking orbits under scaling, the total number of degree $d$ monic polynomials in $n$ variables is

$$
\left|\operatorname{Poly}_{d, n}\left(\mathbb{F}_{q}\right)\right|=\frac{q^{\left(\begin{array}{c}
d+n \\
n
\end{array}\right)}-q^{\left(\begin{array}{c}
d+n-1 \\
n
\end{array}\right)}}{q-1} .
$$

(2) We proceed by induction on $d$ to show that $M_{d, n}(q)$ is a polynomial in $q$. If $d=1$, then all polynomials are irreducible, hence

$$
M_{1, n}(q)=\left|\operatorname{Irr}_{1, n}\left(\mathbb{F}_{q}\right)\right|=\left|\operatorname{Poly}_{1, n}\left(\mathbb{F}_{q}\right)\right|=\frac{q^{n+1}-q}{q-1} .
$$

Suppose our claim is true for all degrees less than $d>1$. By unique factorization, the total number of polynomials with factorization type $\lambda$ is

$$
T_{\lambda, n}(q):=\left|\operatorname{Poly}_{\lambda, n}\left(\mathbb{F}_{q}\right)\right|=\prod_{j \geqslant 1}\left(\left(\begin{array}{c}
M_{j, n}(q) \\
m_{j}(\lambda)
\end{array}\right)\right) .
$$

Counting elements on both sides of the decomposition

$$
\operatorname{Poly}_{d, n}\left(\mathbb{F}_{q}\right)=\bigsqcup_{\lambda \vdash d} \operatorname{Poly}_{\lambda, n}\left(\mathbb{F}_{q}\right),
$$

gives

$$
P_{d, n}(q)=M_{d, n}(q)+\sum_{\substack{\lambda \vdash d \\ \lambda \neq(d)}} T_{\lambda, n}(q)
$$

If $\lambda \neq(d)$, then all parts $j$ of $\lambda$ are smaller than $d$, which by our inductive hypothesis implies that $M_{j, n}(q)$ is a polynomial for all such $j$, hence so is $T_{\lambda, n}(q)$. Thus

$$
M_{d, n}(q)=P_{d, n}(q)-\sum_{\substack{\lambda \vdash d \\ \lambda \neq(d)}} T_{\lambda, n}(q) \in \mathbb{Q}[q] .
$$

Finally, (3) follows from equation (4) and (2) .

The definitions of the polynomials appearing in Lemma 2.1 are collected here for convenience.

DEFINITION 2.2. Let $d, n \geqslant 1$ and $\lambda \vdash d$, then

$$
\begin{aligned}
& P_{d, n}(q):=\frac{q^{\left(\begin{array}{c}
d+n \\
n
\end{array}\right)}-q^{\left(\begin{array}{c}
d+n-1 \\
n
\end{array}\right)}}{q-1}=q^{\left(\begin{array}{c}
d+n-1 \\
n
\end{array}\right)} \frac{q^{\left(\begin{array}{c}
d+n-1 \\
n-1
\end{array}\right)}-1}{q-1} \\
& M_{d, n}(q):=\left|\operatorname{Irr}_{d, n}\left(\mathbb{F}_{q}\right)\right|=\left|\operatorname{Poly}_{(d), n}\left(\mathbb{F}_{q}\right)\right| \\
& T_{\lambda, n}(q):=\left|\operatorname{Poly}_{\lambda, n}\left(\mathbb{F}_{q}\right)\right|=\prod_{j \geqslant 1}\left(\left(\begin{array}{c}
M_{j, n}(q) \\
m_{j}(\lambda)
\end{array}\right)\right) \\
& T_{\lambda, n}^{m}(q):=\left|\operatorname{Poly}_{\lambda, n}^{m}\left(\mathbb{F}_{q}\right)\right| \\
& T_{\lambda, n}^{\mathrm{sf}}(q)=T_{\lambda, n}^{1}(q):=\left|\operatorname{Poly}_{\lambda, n}^{\text {sf }}\left(\mathbb{F}_{q}\right)\right|=\prod_{j \geqslant 1}\left(\begin{array}{c}
M_{j, n}(q) \\
m_{j}(\lambda)
\end{array}\right) \\
& P_{d, n}^{m}(q):=\left|\operatorname{Poly}_{d, n}^{m}\left(\mathbb{F}_{q}\right)\right|=\sum_{\lambda \vdash d} T_{\lambda, n}^{m}(q),
\end{aligned}
$$

where $d$ represents degree, $n$ the number of variables, and $m$ the maximum multiplicity of a factor. 
There is a well-known formula [13, Cor. 2.1] for $M_{d, 1}(q)$ given by counting elements in $\mathbb{F}_{q^{d}}$ by the field they generate,

$$
M_{d, 1}(q)=\frac{1}{d} \sum_{e \mid d} \mu(e) q^{d / e} .
$$

The value of $M_{d, 1}(k)$ for an integer $k \geqslant 1$ has a combinatorial interpretation as the number of aperiodic necklaces made with beads of $k$ colors. For this reason, $M_{d, 1}(q)$ is known as the $d$ th necklace polynomial. There is no apparent analog of (5) nor a necklace interpretation for $M_{d, n}(k)$ when $n>1$. Instead $M_{d, n}(q)$ may be computed recursively as in the proof of Lemma 2.1:

$$
\begin{aligned}
& M_{1, n}(q)=P_{1, n}(q)=\frac{q^{n+1}-q}{q-1} \\
& M_{d, n}(q)=P_{d, n}(q)-\sum_{\substack{\lambda \vdash d \\
\lambda \neq[d]}} T_{\lambda, n}(q) .
\end{aligned}
$$

Our next result shows that all the polynomials listed in Definition 2.2 converge coefficientwise to rational functions in the ring of formal power series $\mathbb{Q} \llbracket q \rrbracket$ as the number of variables $n$ tends to infinity. Recall that coefficientwise convergence in $\mathbb{Q} \llbracket q \rrbracket$ is equivalent to convergence with respect to the $q$-adic topology. All coefficientwise limits are taken with respect to the $q$-adic topology.

THEOREM 2.3. Let $d \geqslant 1$. Then,

(1) The sequence $P_{d, n}(q)$ converges coefficientwise in $\mathbb{Q} \llbracket q \rrbracket$ to

$$
P_{d, \infty}(q)=\lim _{n \rightarrow \infty} P_{d, n}(q)=\left\{\begin{array}{cc}
-\frac{1}{1-\frac{1}{q}} & d=1 \\
0 & d>1 .
\end{array}\right.
$$

(2) For $m \geqslant 1$ the sequence $P_{d, n}^{m}(q)$ converges coefficientwise in $\mathbb{Q} \llbracket q \rrbracket$ to

$$
P_{d, \infty}^{m}(q)=\lim _{n \rightarrow \infty} P_{d, n}^{m}(q)=\left\{\begin{array}{cl}
-\left(\frac{1}{1-\frac{1}{q}}\right)^{k} & d=(m+1) k-m \\
\left(\frac{1}{1-\frac{1}{q}}\right)^{k} & d=(m+1) k \\
0 & d \neq 0,1 \bmod m+1 .
\end{array}\right.
$$

In particular, if $m=1$, then

$$
P_{d, \infty}^{\mathrm{sf}}(q)=(-1)^{d}\left(\frac{1}{1-\frac{1}{q}}\right)^{\left\lfloor\frac{d+1}{2}\right\rfloor} .
$$

(3) For all partitions $\lambda \vdash d$ and $m \geqslant 1$ the sequences $M_{d, n}(q), T_{\lambda, n}(q)$, and $T_{\lambda, n}^{m}(q)$ converge coefficientwise in $\mathbb{Q} \llbracket q \rrbracket$ to rational functions as $n \rightarrow \infty$. Furthermore,

$$
\begin{aligned}
& T_{\lambda, \infty}(q)=\prod_{j \geqslant 1}\left(\left(\begin{array}{c}
M_{j, \infty}(q) \\
m_{j}(\lambda)
\end{array}\right)\right) \\
& T_{\lambda, \infty}^{\mathrm{sf}}(q)=\prod_{j \geqslant 1}\left(\begin{array}{c}
M_{j, \infty}(q) \\
m_{j}(\lambda)
\end{array}\right) .
\end{aligned}
$$

Proof. (1) By Lemma 2.1

$$
P_{d, n}(q)=q^{\left(\begin{array}{c}
d+n-1 \\
n
\end{array}\right)} \frac{q^{\left(\begin{array}{c}
d+n-1 \\
n-1
\end{array}\right)}-1}{q-1} .
$$


For $d=1$ this simplifies to

$$
P_{1, n}(q)=\frac{q^{n+1}-q}{q-1}
$$

Since $\lim _{n \rightarrow \infty} q^{n}=0$ in $\mathbb{Q} \llbracket q \rrbracket$, it follows that

$$
P_{1, \infty}(q)=\lim _{n \rightarrow \infty} \frac{q^{n+1}-q}{q-1}=-\frac{q}{q-1}=-\frac{1}{1-\frac{1}{q}}
$$

If $d>1$, then $\lim _{n \rightarrow \infty}\left(\begin{array}{c}d+n-1 \\ n\end{array}\right)=\infty$. Thus

$$
P_{d, \infty}(q)=\lim _{n \rightarrow \infty} q^{\left(\begin{array}{c}
d+n-1 \\
n
\end{array}\right)} \frac{q^{\left(\begin{array}{c}
d+n-1 \\
n-1
\end{array}\right)}-1}{q-1}=0 .
$$

(2) Consider the generating functions

$$
\begin{aligned}
Z\left(T_{n}^{m}, t\right) & :=\sum_{d \geqslant 0} P_{d, n}^{m}(q) t^{d}=\sum_{d \geqslant 0} \sum_{\lambda \vdash d} T_{\lambda, n}^{m}(q) t^{d} \\
Z\left(T_{n}, t\right) & :=\sum_{d \geqslant 0} P_{d, n}(q) t^{d}=\sum_{d \geqslant 0} \sum_{\lambda \vdash d} T_{\lambda, n}(q) t^{d} .
\end{aligned}
$$

The binomial theorem allows us to formally exponentiate $1+t$ or $\frac{1}{1-t}$ by any element $\alpha \in R$ of a binomial ring $^{(2)}$ in $R \llbracket t \rrbracket$ by

$$
\begin{gathered}
(1+t)^{\alpha}:=\sum_{d \geqslant 0}\left(\begin{array}{l}
\alpha \\
d
\end{array}\right) t^{d}, \\
\left(\frac{1}{1-t}\right)^{\alpha}:=\sum_{d \geqslant 0}\left(\left(\begin{array}{l}
\alpha \\
d
\end{array}\right)\right) t^{d} .
\end{gathered}
$$

The following product formulas follow by unique factorization in $\mathbb{F}_{q}\left[x_{1}, x_{2}, \ldots, x_{n}\right]$,

$$
\begin{aligned}
& Z\left(T_{n}^{m}, t\right)=\prod_{j \geqslant 1}\left(1+t^{j}+t^{2 j}+\ldots+t^{m j}\right)^{M_{j, n}(q)}=\prod_{j \geqslant 1}\left(\frac{1-t^{(m+1) j}}{1-t^{j}}\right)^{M_{j, n}(q)} \\
& Z\left(T_{n}, t\right)=\prod_{j \geqslant 1}\left(\frac{1}{1-t^{j}}\right)^{M_{j, n}(q)} .
\end{aligned}
$$

Hence $Z\left(T_{n}, t\right)=Z\left(T_{n}, t^{m+1}\right) Z\left(T_{n}^{m}, t\right)$. The coefficients of $t^{d}$ for $d \geqslant 0$ in this identity are polynomials which converge $q$-adically in $\mathbb{Q} \llbracket q \rrbracket$ as $n \rightarrow \infty$. Taking a limit $t$ coefficientwise as $n \rightarrow \infty$, (1) implies that

$$
1-\frac{1}{1-\frac{1}{q}} t=Z\left(T_{\infty}, t\right)=Z\left(T_{\infty}, t^{m+1}\right) Z\left(T_{\infty}^{m}, t\right)=\left(1-\frac{1}{1-\frac{1}{q}} t^{m+1}\right) \sum_{d \geqslant 0} P_{d, \infty}^{m}(q) t^{d} .
$$

Comparing coefficients we conclude that

$$
P_{d+m+1, \infty}^{m}(q)=\frac{1}{1-\frac{1}{q}} P_{d, \infty}^{m}(q)
$$

${ }^{(2)} \mathrm{A}$ binomial ring $R$ is a commutative ring with no additive torsion which is closed under taking binomial coefficients. 
for all $d \geqslant 0$, together with the initial values

$$
\begin{aligned}
& P_{0, \infty}^{m}(q)=1 \\
& P_{1, \infty}^{m}(q)=-\frac{1}{1-\frac{1}{q}} \\
& P_{d, \infty}^{m}(q)=0 \text { for } 1<d \leqslant m .
\end{aligned}
$$

Then (2) follows by induction.

(3) It suffices to prove that for every $d \geqslant 1$ the sequence $M_{d, n}(q)$ converges $q$-adically to a rational function, the other claims follow by the explicit formulas given in Definition 2.2 and continuity. Recall the recursive formulas for $M_{d, n}(q)$ used in the proof of Lemma 2.1. For all $d, n \geqslant 1$,

$$
\begin{aligned}
& M_{1, n}(q)=P_{1, n}(q) \\
& M_{d, n}(q)=P_{d, n}(q)-\sum_{\substack{\lambda \vdash d \\
\lambda \neq[d]}} \prod_{j \geqslant 1}\left(\left(\begin{array}{c}
M_{j, n}(q) \\
m_{j}(\lambda)
\end{array}\right)\right) .
\end{aligned}
$$

Taking coefficientwise limits as $n \rightarrow \infty$ using (1) we have

$$
\begin{aligned}
& M_{1, \infty}(q)=P_{1, \infty}(q)=-\frac{1}{1-\frac{1}{q}}, \\
& M_{d, \infty}(q)=-\sum_{\substack{\lambda \vdash d \\
\lambda \neq[d]}} \prod_{j \geqslant 1}\left(\left(\begin{array}{c}
M_{j, \infty}(q) \\
m_{j}(\lambda)
\end{array}\right)\right) .
\end{aligned}
$$

It follows by induction that $M_{d, \infty}(q)$ is a rational function of $q$ for all $d \geqslant 1$.

There is a surprising relationship between the number of irreducible polynomials in one variable $M_{d, 1}(q)$ and the limit $M_{d, \infty}(q)$ of the number of irreducible polynomials in $n$ variables as $n \rightarrow \infty$, which gives us an explicit formula for $M_{d, \infty}(q)$. This relationship takes the form of an involutive functional equation we call liminal reciprocity.

Theorem 2.4 (Liminal reciprocity). For all $d \geqslant 1$,

$$
M_{d, \infty}(q)=-M_{d, 1}\left(\frac{1}{1-\frac{1}{q}}\right) .
$$

More explicitly,

$$
M_{d, \infty}(q)=-\frac{1}{d} \sum_{e \mid d} \mu(e)\left(\frac{1}{1-\frac{1}{q}}\right)^{d / e} .
$$

We make use of the following well-known lemma. A proof can be found, for example, in [9, Lem. 4.2].

Lemma 2.5. For any binomial ring $R$ and any sequence $a_{d} \in R$ for $d \geqslant 0$ such that $a_{0}=1$ there exists a unique sequence $b_{j} \in R$ for $j \geqslant 1$ such that the following identity holds in $R \llbracket t \rrbracket$.

$$
\sum_{d \geqslant 0} a_{d} t^{d}=\prod_{j \geqslant 1}\left(\frac{1}{1-t^{j}}\right)^{b_{j}} .
$$


Proof. Recall the generating function $Z\left(T_{n}, t\right)$ used in the proof of Theorem $2.3(2)$,

$$
Z\left(T_{n}, t\right)=\sum_{d \geqslant 0} P_{d, n}(q) t^{d}=\prod_{j \geqslant 1}\left(\frac{1}{1-t^{j}}\right)^{M_{j, n}(q)}
$$

Theorem 2.3 (1) implies that the $t$-coefficientwise limit as $n \rightarrow \infty$ is simply

$$
1-\frac{1}{1-\frac{1}{q}} t=\prod_{d \geqslant 1}\left(\frac{1}{1-t^{d}}\right)^{M_{d, \infty}(q)} .
$$

When $n=1, P_{d, 1}(q)=q^{d}$ and thus

$$
\frac{1}{1-q t}=Z\left(T_{1}, t\right)=\prod_{d \geqslant 1}\left(\frac{1}{1-t^{d}}\right)^{M_{d, 1}(q)} .
$$

Substituting $q \mapsto \frac{1}{1-\frac{1}{q}}$ and taking reciprocals in (8) gives

$$
1-\frac{1}{1-\frac{1}{q}} t=\prod_{d \geqslant 1}\left(\frac{1}{1-t^{d}}\right)^{-M_{d, 1}\left(\frac{1}{1-\frac{1}{q}}\right)} .
$$

Comparing exponents with (7) and using the uniqueness of Lemma 2.5 we conclude that

$$
M_{d, \infty}(q)=-M_{d, 1}\left(\frac{1}{1-\frac{1}{q}}\right) .
$$

REMARK 2.6. The identity (8) is known as the cyclotomic identity [12]. It also arises as the Euler product formula for the Hasse-Weil zeta function of $\mathbb{A}^{1}\left(\mathbb{F}_{q}\right)$.

The rate of $q$-adic convergence of $M_{d, n}(q)$ may be determined from the proof of Theorem 2.4.

Corollary 2.7. For all $d, n \geqslant 1$,

$$
M_{d, n}(q) \equiv M_{d, \infty}(q) \bmod q^{n+1}
$$

Proof. Recall that

$$
P_{d, n}(q)=q^{\left(\begin{array}{c}
d+n-1 \\
n
\end{array}\right)} \frac{q^{\left(\begin{array}{c}
d+n-1 \\
n-1
\end{array}\right)}-1}{q-1} .
$$

Since $\left(\begin{array}{c}d+n-1 \\ n\end{array}\right) \geqslant n+1$ for $d \geqslant 2$ and

$$
P_{1, n}(q)=\frac{q^{n+1}-q}{q-1} \equiv-\frac{1}{1-\frac{1}{q}} \bmod q^{n+1},
$$

it follows that

$$
\sum_{d \geqslant 0} P_{d, n}(q) t^{d} \equiv 1-\frac{1}{1-\frac{1}{q}} t \bmod q^{n+1}
$$

Thus

$$
\begin{aligned}
\prod_{d \geqslant 1}\left(\frac{1}{1-t^{d}}\right)^{M_{d, n}(q)} & =\sum_{d \geqslant 0} P_{d, n}(q) t^{d} \\
& \equiv 1-\frac{1}{1-\frac{1}{q}} t \bmod q^{n+1} \\
& \equiv \prod_{d \geqslant 1}\left(\frac{1}{1-t^{d}}\right)^{M_{d, \infty}(q)} \bmod q^{n+1}
\end{aligned}
$$


Therefore by Lemma 2.5

$$
M_{d, n}(q) \equiv M_{d, \infty}(q) \bmod q^{n+1} .
$$

REMARK 2.8. Notice that the fractional linear transformation $q \longmapsto \frac{1}{1-\frac{1}{q}}$ is an involution. Thus Theorem 2.4 is equivalent to

$$
M_{d, 1}(q)=-M_{d, \infty}\left(\frac{1}{1-\frac{1}{q}}\right) .
$$

This is the sense in which we consider Theorem 2.4 a "reciprocity."

Our next result combines Theorem 2.4 with the combinatorial reciprocity identity

$$
\left(\begin{array}{c}
-x \\
m
\end{array}\right)=(-1)^{m}\left(\left(\begin{array}{c}
x \\
m
\end{array}\right)\right),
$$

to deduce a striking relationship between factorization statistics of polynomials when $n=1$ and $n=\infty$.

THEOREM 2.9 (Liminal reciprocity). For any partition $\lambda$, let $\ell(\lambda)=\sum_{j \geqslant 1} m_{j}(\lambda)$ denote the number of parts of $\lambda$. Then

$$
\begin{aligned}
& T_{\lambda, \infty}^{\mathrm{sf}}(q)=(-1)^{\ell(\lambda)} T_{\lambda, 1}\left(\frac{1}{1-\frac{1}{q}}\right), \\
& T_{\lambda, \infty}(q)=(-1)^{\ell(\lambda)} T_{\lambda, 1}^{\mathrm{sf}}\left(\frac{1}{1-\frac{1}{q}}\right) .
\end{aligned}
$$

Proof. Theorem 2.3 (3), Theorem 2.4, and the combinatorial reciprocity identity (9) imply that

$$
\begin{aligned}
T_{\lambda, \infty}^{\mathrm{sf}}(q) & =\prod_{j \geqslant 1}\left(\begin{array}{c}
M_{j, \infty}(q) \\
m_{j}(\lambda)
\end{array}\right) \\
& =\prod_{j \geqslant 1}\left(\begin{array}{c}
-M_{j, 1}\left(\frac{1}{1-\frac{1}{q}}\right) \\
m_{j}(\lambda)
\end{array}\right) \\
& =\prod_{j \geqslant 1}(-1)^{m_{j}(\lambda)}\left(\left(\begin{array}{c}
M_{j, 1}\left(\frac{1}{1-\frac{1}{q}}\right) \\
m_{j}(\lambda)
\end{array}\right)\right) \\
& =(-1)^{\ell(\lambda)} T_{\lambda, 1}\left(\frac{1}{1-\frac{1}{q}}\right) .
\end{aligned}
$$

The second identity follows from a parallel computation noting that (9) is equivalent to

$$
\left(\left(\begin{array}{c}
-x \\
m
\end{array}\right)\right)=(-1)^{m}\left(\begin{array}{c}
x \\
m
\end{array}\right) .
$$

The liminal reciprocity identity

$$
T_{\lambda, \infty}^{\mathrm{sf}}(q)=(-1)^{\ell(\lambda)} T_{\lambda, 1}\left(\frac{1}{1-\frac{1}{q}}\right)
$$

relates the limiting number of squarefree polynomials with factorization type $\lambda$ in $\mathbb{F}_{q}\left[x_{1}, x_{2}, \ldots, x_{n}\right]$ as $n \rightarrow \infty$ to the number of polynomials $\mathbb{F}_{q}[x]$ with factorization type $\lambda$ with no restrictions on factor multiplicity. This relationship is, to us, mysterious. It would be interesting to find a conceptual explanation for this relationship between infinite and one dimensional factorization statistics. 


\section{LIMINAL FIRST MOMENTS OF SQUAREFREE FACTORIZATION STATISTICS}

A factorization statistic is a function $P$ defined on $\operatorname{Poly}_{d, n}\left(\mathbb{F}_{q}\right)$ such that $P(f)$ only depends on the factorization type of $f \in \operatorname{Poly}_{d, n}\left(\mathbb{F}_{q}\right)$. Equivalently, $P$ is a function defined on the partitions of the degree $d$, or a class function of the symmetric group $S_{d}$. In [10] we determined explicit formulas for the first moments of factorization statistics on $\operatorname{Poly}_{d, 1}\left(\mathbb{F}_{q}\right)$ and Poly $_{d, 1}^{\mathrm{sf}}\left(\mathbb{F}_{q}\right)$ in terms of the characters of symmetric group representations carried by the cohomology of point configurations in Euclidean space.

If $X$ is a topological space, then the ordered configuration space of $d$ points in $X$ is defined as

$$
\operatorname{PConf}_{d}(X):=\left\{\left(a_{1}, a_{2}, \ldots, a_{d}\right) \in X^{d}: a_{i} \neq a_{j}\right\} .
$$

The symmetric group $S_{d}$ acts on $\mathrm{PConf}_{d}(X)$ by permuting the labels of points, and thus the singular cohomology $H^{k}\left(\operatorname{PConf}_{d}(X), \mathbb{Q}\right)$ is a linear representation of $S_{d}$ for each cohomological degree $k$.

Theorem 3.1 ([10, Thm. 2.2, Thm. 2.3], [6, Prop. 4.1]). Let $P$ be a factorization statistic, and let $\psi_{d}^{k}, \phi_{d}^{k}$ be the characters of the $S_{d}$-representations $H^{2 k}\left(\operatorname{PConf}_{d}\left(\mathbb{R}^{3}\right), \mathbb{Q}\right)$ and $H^{k}\left(\mathrm{PConf}_{d}\left(\mathbb{R}^{2}\right), \mathbb{Q}\right)$ respectively. Then

$$
\begin{aligned}
\sum_{f \in \operatorname{Poly}_{d, 1}\left(\mathbb{F}_{q}\right)} P(f) & =\sum_{k=0}^{d-1}\left\langle P, \psi_{d}^{k}\right\rangle q^{d-k} \\
\sum_{f \in \operatorname{Poly}_{d, 1}^{\text {sf }}\left(\mathbb{F}_{q}\right)} P(f) & =\sum_{k=0}^{d-1}(-1)^{k}\left\langle P, \phi_{d}^{k}\right\rangle q^{d-k},
\end{aligned}
$$

where $\langle P, Q\rangle=\frac{1}{d !} \sum_{\tau \in S_{d}} P(\tau) Q(\tau)$ is the standard inner product of class functions of $S_{d}$.

The identity (2) was first shown by Church, Ellenberg, and Farb [6, Prop. 4.1] using algebro-geometric methods including the Grothendieck-Lefschetz trace formula. They called this identity the twisted Grothendieck-Lefschetz formula for squarefree polynomials. We gave a new proof in [10, Thm. 2.3] using a generating function argument. Our results in [10] are stated in terms of expected values instead of first moments; this distinction has little effect in the $\operatorname{Poly}_{d, 1}\left(\mathbb{F}_{q}\right)$ case, but does change the family of representations in the squarefree case $\operatorname{Poly}_{d, 1}^{\text {sf }}\left(\mathbb{F}_{q}\right)$; since the total number of degree $d$ squarefree polynomials in $\mathbb{F}_{q}[x]$ is $P_{d, 1}^{\mathrm{sf}}(q)=q^{d}-q^{d-1}$, dividing the first moment of a factorization statistic by $q^{d}-q^{d-1}$ results in a polynomial with different coefficients. This version of (2) appears in [6, Prop. 4.1].

Our next result combines Theorem 3.1 with liminal reciprocity to express the limiting first moments of squarefree factorization statistics in terms of characters of symmetric group representations.

TheOrem 3.2. Let $P$ be a factorization statistic, and let $\sigma_{d}^{k}$ be the character of the $S_{d}$-representation

$$
\Sigma_{d}^{k}=\bigoplus_{j=k}^{d-1} \operatorname{sgn}_{d} \otimes H^{2 j}\left(\operatorname{PConf}_{d}\left(\mathbb{R}^{3}\right), \mathbb{Q}\right)^{\oplus\left(\begin{array}{l}
j \\
k
\end{array}\right)} .
$$

Then

$$
\lim _{n \rightarrow \infty} \sum_{f \in \mathrm{Poly}_{d, n}^{\mathrm{sf}}\left(\mathbb{F}_{q}\right)} P(f)=\frac{1}{(1-q)^{d}} \sum_{k=0}^{d}(-1)^{k}\left\langle P, \sigma_{d}^{k}\right\rangle q^{d-k}
$$


Theorem 3.2 follows from the following representation theoretic interpretation of the liminal squarefree type polynomials $T_{\lambda, \infty}^{\text {sf }}(q)$. Recall that for a partition $\lambda$ the liminal squarefree type polynomial $T_{\lambda, \infty}^{\mathrm{sf}}(q)$ is defined by

$$
T_{\lambda, \infty}^{\mathrm{sf}}(q):=\lim _{n \rightarrow \infty} T_{\lambda, n}^{\mathrm{sf}}(q)
$$

where $T_{\lambda, n}^{\mathrm{sf}}(q)$ is the number of monic squarefree polynomials in $\mathbb{F}_{q}\left[x_{1}, x_{2}, \ldots, x_{n}\right]$ with factorization type $\lambda$.

THEOREM 3.3. Let $\lambda \vdash d$ be a partition, and let $\sigma_{d}^{k}$ be the character of the $S_{d^{-}}$ representation $\Sigma_{d}^{k}$ defined in (10). Then

$$
T_{\lambda, \infty}^{\mathrm{sf}}(q)=\frac{1}{z_{\lambda}(1-q)^{d}} \sum_{k=0}^{d-1}(-1)^{k} \sigma_{d}^{k}(\lambda) q^{d-k},
$$

where $z_{\lambda}:=\prod_{j \geqslant 1} j^{m_{j}(\lambda)} m_{j}(\lambda)$ ! is the number of permutations in $S_{d}$ commuting with a permutation of cycle type $\lambda$.

Proof. Let $\psi_{d}^{k}$ be the character of the $S_{d}$-representation $H^{2 k}\left(\operatorname{PConf}_{d}\left(\mathbb{R}^{3}\right), \mathbb{Q}\right)$. In $[10$, Thm. 2.1] we showed that for all partitions $\lambda \vdash d$,

$$
T_{\lambda, 1}(q)=\frac{1}{z_{\lambda}} \sum_{k=0}^{d-1} \psi_{d}^{k}(\lambda) q^{d-k}
$$

Thus, Theorem 2.9 gives

$$
\begin{aligned}
T_{\lambda, \infty}^{\mathrm{sf}}(q) & =(-1)^{\ell(\lambda)} T_{\lambda, 1}\left(\frac{1}{1-\frac{1}{q}}\right) \\
& =\frac{1}{z_{\lambda}} \sum_{j=0}^{d-1}(-1)^{\ell(\lambda)} \psi_{d}^{j}(\lambda)\left(\frac{1}{1-\frac{1}{q}}\right)^{d-j} \\
& =\frac{1}{z_{\lambda}(1-q)^{d}} \sum_{j=0}^{d-1}(-1)^{d-\ell(\lambda)} \psi_{d}^{j}(\lambda) q^{d-j}(q-1)^{j} \\
& =\frac{1}{z_{\lambda}(1-q)^{d}} \sum_{j=0}^{d-1} \operatorname{sgn}_{d}(\lambda) \psi_{d}^{j}(\lambda) q^{d-j} \sum_{k=0}^{j}(-1)^{k}\left(\begin{array}{l}
j \\
k
\end{array}\right) q^{j-k} \\
& =\frac{1}{z_{\lambda}(1-q)^{d}} \sum_{k=0}^{d-1}(-1)^{k}\left(\sum_{j=k}^{d}\left(\begin{array}{l}
j \\
k
\end{array}\right) \operatorname{sgn}_{d}(\lambda) \psi_{d}^{j}(\lambda)\right) q^{d-k} \\
& =\frac{1}{z_{\lambda}(1-q)^{d}} \sum_{k=0}^{d-1}(-1)^{k} \sigma_{d}^{k}(\lambda) q^{d-k} .
\end{aligned}
$$

We now prove Theorem 3.3.

Proof. Since $P$ depends only on factorization type, the limiting first moment of $P$ may be rewritten as

$$
\lim _{n \rightarrow \infty} \sum_{f \in \operatorname{Poly}_{d, n}^{\mathrm{sf}}\left(\mathbb{F}_{q}\right)} P(f)=\lim _{n \rightarrow \infty} \sum_{\lambda \vdash d} P(\lambda) T_{\lambda, n}^{\mathrm{sf}}(q)=\sum_{\lambda \vdash d} P(\lambda) T_{\lambda, \infty}^{\mathrm{sf}}(q) .
$$


Then Theorem 3.3 implies

$$
\begin{aligned}
\sum_{\lambda \vdash d} P(\lambda) T_{\lambda, \infty}^{\mathrm{sf}}(q) & =\sum_{\lambda \vdash d} \frac{1}{z_{\lambda}(1-q)^{d}} \sum_{k=0}^{d-1}(-1)^{k} P(\lambda) \sigma_{d}^{k}(\lambda) q^{d-k} \\
& =\frac{1}{(1-q)^{d}} \sum_{k=0}^{d-1}(-1)^{k} \sum_{\lambda \vdash d} \frac{P(\lambda) \sigma_{d}^{k}(\lambda)}{z_{\lambda}} q^{d-k} \\
& =\frac{1}{(1-q)^{d}} \sum_{k=0}^{d-1}(-1)^{k}\left\langle P, \sigma_{d}^{k}\right\rangle q^{d-k}
\end{aligned}
$$

The coefficients of $T_{\lambda, 1}^{\mathrm{sf}}(q)$ also have representation theoretic interpretations, which suggests that we might hope for a version of Theorem 3.3 for the limiting first moments of factorization statistics on $\operatorname{Poly}_{d, n}\left(\mathbb{F}_{q}\right)$. However, computations show that the coefficients of $T_{\lambda, \infty}(q)$ are determined by virtual characters, unlike those of $T_{\lambda, \infty}^{\mathrm{sf}}(q)$.

In [10] we pose the question of finding a geometric interpretation of Theorem 3.1 which explains the connection between the configuration space PConf ${ }_{d}\left(\mathbb{R}^{3}\right)$ and factorization statistics of degree $d$ polynomials over $\mathbb{F}_{q}$. Furthermore, we would like a conceptual interpretation of Theorem 3.3, be it geometric or combinatorial. The family of representations $\Sigma_{d}^{k}$ is unfamiliar to us; we collect some of their basic properties in Proposition 3.5.

3.1. ExAmple. We demonstrate the liminal reciprocity identity of Theorem 2.9 by computing the expected value of the sign statistic $\operatorname{sgn}_{d}$ on degree $d$ univariate polynomials $\operatorname{Poly}_{d, 1}\left(\mathbb{F}_{q}\right)$ and the limiting expected value of $\operatorname{sgn}_{d}$ on squarefree degree $d$ polynomials $\operatorname{Poly}_{d, \infty}^{\mathrm{sf}}\left(\mathbb{F}_{q}\right)$.

Let $\operatorname{sgn}_{d}$ be the sign character of $S_{d}$. Note that $\operatorname{sgn}_{d}(\lambda)=(-1)^{d}(-1)^{\ell(\lambda)}$, where $\ell(\lambda)=\sum_{j \geqslant 1} m_{j}(\lambda)$ is the number of parts of $\lambda$. Recall that $P_{d, n}(q)=\left|\operatorname{Poly}_{d, n}\left(\mathbb{F}_{q}\right)\right|$ and $P_{d, n}^{\text {sf }}(q)=\left|\operatorname{Poly}_{d, n}^{\mathrm{sf}}\left(\mathbb{F}_{q}\right)\right|$.

Proposition 3.4. Let $d \geqslant 1$.

(1) The expected value $E_{d, 1}\left(\operatorname{sgn}_{d}\right)$ of the sign statistic on the set $\operatorname{Poly}_{d, 1}\left(\mathbb{F}_{q}\right)$ is given by

$$
E_{d, 1}\left(\operatorname{sgn}_{d}\right):=\frac{1}{P_{d, 1}(q)} \sum_{f \in \operatorname{Poly}_{d, 1}\left(\mathbb{F}_{q}\right)} \operatorname{sgn}_{d}(f)=\frac{1}{q^{\lfloor d / 2\rfloor}} .
$$

(2) The limiting expected value $E_{d, \infty}^{\mathrm{sf}}\left(\operatorname{sgn}_{d}\right)$ of the sign statistic on the set Poly $_{d, n}^{\mathrm{sf}}\left(\mathbb{F}_{q}\right)$ as $n \rightarrow \infty$ is given by

$$
E_{d, \infty}^{\mathrm{sf}}\left(\operatorname{sgn}_{d}\right):=\lim _{n \rightarrow \infty} \frac{1}{P_{d, n}^{\mathrm{sf}}(q)} \sum_{f \in \operatorname{Poly}_{d, n}^{\mathrm{sf}}\left(\mathbb{F}_{q}\right)} \operatorname{sgn}_{d}(f)=\left(\frac{1}{1-\frac{1}{q}}\right)^{\lfloor d / 2\rfloor},
$$

where the limit is taken q-adically.

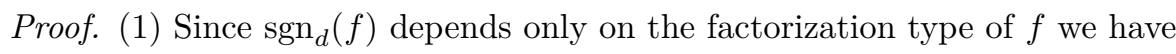

$$
\sum_{f \in \text { Poly }_{d, 1}\left(\mathbb{F}_{q}\right)} \operatorname{sgn}_{d}(f)=\sum_{\lambda \vdash d} \operatorname{sgn}(\lambda) T_{\lambda, 1}(q) .
$$

Theorem 2.9 gives the identity

$$
(-1)^{\ell(\lambda)} T_{\lambda, 1}(q)=T_{\lambda, \infty}^{\mathrm{sf}}\left(\frac{1}{1-\frac{1}{q}}\right)
$$


from which we deduce for each $d \geqslant 1$

$$
\begin{aligned}
\sum_{\lambda \vdash d} \operatorname{sgn}(\lambda) T_{\lambda, 1}(q) & =\sum_{\lambda \vdash d}(-1)^{d}(-1)^{\ell(\lambda)} T_{\lambda, 1}(q) \\
& =\sum_{\lambda \vdash d}(-1)^{d} T_{\lambda, \infty}^{\mathrm{sf}}\left(\frac{1}{1-\frac{1}{q}}\right) \\
& =(-1)^{d} P_{d, \infty}^{\mathrm{sf}}\left(\frac{1}{1-\frac{1}{q}}\right) .
\end{aligned}
$$

Theorem 2.3 (2) tells us

$$
P_{d, \infty}^{\mathrm{sf}}(q)=(-1)^{d}\left(\frac{1}{1-\frac{1}{q}}\right)^{\left\lfloor\frac{d+1}{2}\right\rfloor}
$$

Thus,

$$
\sum_{\lambda \vdash d} \operatorname{sgn}_{d}(\lambda) T_{\lambda, 1}(q)=(-1)^{d} P_{d, \infty}^{\mathrm{sf}}\left(\frac{1}{1-\frac{1}{q}}\right)=q^{\left\lfloor\frac{d+1}{2}\right\rfloor} .
$$

Since $P_{d, 1}(q)=q^{d}$ and $d-\lfloor(d+1) / 2\rfloor=\lfloor d / 2\rfloor$ it follows that

$$
E_{d, 1}\left(\operatorname{sgn}_{d}\right)=\frac{1}{P_{d, 1}(q)} \sum_{f \in \operatorname{Poly}_{d, 1}\left(\mathbb{F}_{q}\right)} \operatorname{sgn}(f)=\frac{1}{q^{\lfloor d / 2\rfloor}} .
$$

(2) For each $n \geqslant 1$,

$$
E_{d, n}^{\mathrm{sf}}\left(\operatorname{sgn}_{d}\right):=\frac{1}{P_{d, n}^{\mathrm{sf}}(q)} \sum_{f \in \operatorname{Poly}_{d, n}^{\mathrm{sf}}\left(\mathbb{F}_{q}\right)} \operatorname{sgn}_{d}(f)=\frac{1}{P_{d, n}^{\mathrm{sf}}(q)} \sum_{\lambda \vdash d} \operatorname{sgn}(\lambda) T_{\lambda, n}^{\mathrm{sf}}(q) .
$$

Taking a limit as $n \rightarrow \infty$,

$$
E_{d, \infty}^{\mathrm{sf}}\left(\operatorname{sgn}_{d}\right)=\frac{1}{P_{d, \infty}^{\mathrm{sf}}(q)} \sum_{\lambda \vdash d} \operatorname{sgn}_{d}(\lambda) T_{\lambda, \infty}^{\mathrm{sf}}(q) .
$$

Theorem 2.9 gives us

$$
(-1)^{\ell(\lambda)} T_{\lambda, \infty}^{\mathrm{sf}}(q)=T_{\lambda, 1}\left(\frac{1}{1-\frac{1}{q}}\right)
$$

Therefore,

$$
\begin{aligned}
\sum_{\lambda \vdash d} \operatorname{sgn}_{d}(\lambda) T_{\lambda, \infty}^{\mathrm{sf}}(q) & =\sum_{\lambda \vdash d}(-1)^{d}(-1)^{\ell(\lambda)} T_{\lambda, \infty}^{\mathrm{sf}}(q) \\
& =\sum_{\lambda \vdash d}(-1)^{d} T_{\lambda, 1}\left(\frac{1}{1-\frac{1}{q}}\right) \\
& =(-1)^{d}\left(\frac{1}{1-\frac{1}{q}}\right)^{d} .
\end{aligned}
$$

Since $P_{d, \infty}^{\mathrm{sf}}(q)=(-1)^{d}\left(\frac{1}{1-\frac{1}{q}}\right)^{\lfloor(d+1) / 2\rfloor}$ and $d-\lfloor(d+1) / 2\rfloor=\lfloor d / 2\rfloor$ we conclude that

$$
E_{d, \infty}^{\mathrm{sf}}\left(\operatorname{sgn}_{d}\right)=\frac{1}{P_{d, \infty}^{\mathrm{sf}}(q)} \sum_{\lambda \vdash d} \operatorname{sgn}_{d}(\lambda) T_{\lambda, \infty}^{\mathrm{sf}}(q)=\left(\frac{1}{1-\frac{1}{q}}\right)^{\lfloor d / 2\rfloor} .
$$


Combining Theorem 3.1 (1) and Proposition 3.4 (1) we find that

$$
E_{d, 1}\left(\operatorname{sgn}_{d}\right)=\sum_{k=0}^{d-1} \frac{\left\langle\operatorname{sgn}_{d}, \psi_{d}^{k}\right\rangle}{q^{k}}=\frac{1}{q^{\lfloor d / 2\rfloor}} .
$$

Therefore it follows that $H^{2 k}\left(\operatorname{PConf}_{d}\left(\mathbb{R}^{3}\right), \mathbb{Q}\right)$ has a one dimensional sgn ${ }_{d}$ component if and only if $k=\lfloor d / 2\rfloor$.

The sign function $\operatorname{sgn}_{d}$ is closely related to the Liouville function $\lambda$ studied by Carlitz $[2,3]$ in the context of polynomials in $\mathbb{F}_{q}[x]$. In particular, if $f(x) \in \operatorname{Poly}_{d, 1}\left(\mathbb{F}_{q}\right)$

$$
\lambda(f)=(-1)^{d} \operatorname{sgn}_{d}(f) .
$$

Carlitz [2, (ii) pg. 121][3, Sec. 3] computes the first moment of the Liouville function using zeta functions. Proposition 3.4 may also be deduced from his result. We thank Ofir Gorodetsky for bringing this work to our attention.

3.2. The $S_{d}$-REpresentations $\Sigma_{d}^{k}$. Theorem 3.2 relates the limiting first moments of factorization statistics on squarefree polynomials with a family of symmetric group representations $\Sigma_{d}^{k}$. Recall that

$$
\Sigma_{d}^{k}:=\bigoplus_{j=k}^{d-1} \operatorname{sgn}_{d} \otimes H^{2 j}\left(\operatorname{PConf}_{d}\left(\mathbb{R}^{3}\right), \mathbb{Q}\right)^{\oplus\left(\begin{array}{l}
j \\
k
\end{array}\right) .}
$$

We conclude with Proposition 3.5 which records some observations about the representations $\Sigma_{d}^{k}$.

Proposition 3.5. Let $\sigma_{d}^{k}$ be the character of $\Sigma_{d}^{k}$. Then

(1) The dimension of $\Sigma_{d}^{k}$ is

$$
\operatorname{dim} \Sigma_{d}^{k}=\sum_{i=k}^{d-1}\left[\begin{array}{c}
d \\
d-i
\end{array}\right]\left(\begin{array}{c}
i \\
i-k
\end{array}\right)
$$

where $\left[\begin{array}{l}m \\ n\end{array}\right]$ is an unsigned Stirling number of the first kind (see below for a definition.)

(2) The representation

$$
\bigoplus_{k=0}^{d-1} \Sigma_{d}^{k}
$$

has dimension $(2 d-1) ! !:=(2 d-1)(2 d-3) \cdots 3 \cdot 1$.

(3) $\Sigma_{d}^{0}$ is isomorphic to the regular representation $\mathbb{Q}\left[S_{d}\right]$.

REMARK 3.6. The sequence $\operatorname{dim} \Sigma_{d}^{k}$ appears as A088996 in the Online Encyclopedia of Integer Sequences [14].

Proof. (1) The dimension of a representation is given by evaluating its character on the identity, hence

Theorem 3.3 implies that

$$
\operatorname{dim} \Sigma_{d}^{k}=\sigma_{d}^{k}\left(\left(1^{d}\right)\right)
$$

$$
T_{\left(1^{d}\right), \infty}^{\mathrm{sf}}(q)=\frac{1}{d !(1-q)^{d}} \sum_{k=0}^{d-1}(-1)^{k} \sigma_{d}^{k}\left(\left(1^{d}\right)\right) q^{d-k} .
$$

On the other hand, we may compute $T_{\left(1^{d}\right), \infty}^{\mathrm{sf}}(q)$ directly as

$$
T_{\left(1^{d}\right), \infty}^{\mathrm{sf}}(q)=\left(\begin{array}{c}
M_{d, \infty}(q) \\
d
\end{array}\right)=\left(\begin{array}{c}
-\frac{1}{1-\frac{1}{q}} \\
d
\end{array}\right) .
$$


The unsigned Stirling numbers of the first kind are defined as the coefficients in the expansion of a binomial coeffcient $\left(\begin{array}{l}x \\ d\end{array}\right)$,

$$
\left(\begin{array}{l}
x \\
d
\end{array}\right)=\frac{1}{d !} \sum_{k=0}^{d-1}(-1)^{k}\left[\begin{array}{c}
d \\
d-k
\end{array}\right] x^{d-k}
$$

Thus,

$$
\begin{aligned}
T_{\left(1^{d}\right), \infty}^{\mathrm{sf}}(q) & =\frac{1}{d !} \sum_{i=0}^{d-1}(-1)^{i}\left[\begin{array}{c}
d \\
d-i
\end{array}\right]\left(-\frac{1}{1-\frac{1}{q}}\right)^{d-i} \\
& =\frac{1}{d !(1-q)^{d}} \sum_{i=0}^{d-1}(-1)^{i}\left[\begin{array}{c}
d \\
d-i
\end{array}\right] q^{d-i}(1-q)^{i} \\
& =\frac{1}{d !(1-q)^{d}} \sum_{i=0}^{d-1} \sum_{j=0}^{i}(-1)^{i+j}\left[\begin{array}{c}
d \\
d-i
\end{array}\right]\left(\begin{array}{c}
i \\
j
\end{array}\right) q^{d-(i-j)}
\end{aligned}
$$

Let $k=i-j$ and write the sum in terms of $i$ and $k$ to get

$$
T_{\left(1^{d}\right), \infty}^{\mathrm{sf}}(q)=\frac{1}{d !(1-q)^{d}} \sum_{k=0}^{d-1}(-1)^{k}\left(\sum_{i=k}^{d-1}\left[\begin{array}{c}
d \\
d-i
\end{array}\right]\left(\begin{array}{c}
i \\
i-k
\end{array}\right)\right) q^{d-k}
$$

Comparing coefficients in our two expressions for $T_{\left(1^{d}\right), \infty}^{\mathrm{sf}}(q)$ we conclude that

$$
\operatorname{dim} \Sigma_{d}^{k}=\sigma_{d}^{k}\left(\left(1^{d}\right)\right)=\sum_{i=k}^{d-1}\left[\begin{array}{c}
d \\
d-i
\end{array}\right]\left(\begin{array}{c}
i \\
i-k
\end{array}\right)
$$

(2) Let $\psi_{d}^{k}$ be the character of $H^{2 k}\left(\operatorname{PConf}_{d}\left(\mathbb{R}^{3}\right), \mathbb{Q}\right)$. Then using the definition of $\Sigma_{d}^{k}$ and switching the order of summation we have

$$
\sum_{k=0}^{d-1} \sigma_{d}^{k}\left(\left(1^{d}\right)\right)=\sum_{k=0}^{d-1} \sum_{j=k}^{d}\left(\begin{array}{l}
j \\
k
\end{array}\right) \psi_{d}^{j}\left(\left(1^{d}\right)\right)=\sum_{j=0}^{d-1} \sum_{k=0}^{j}\left(\begin{array}{l}
j \\
k
\end{array}\right) \psi_{d}^{j}\left(\left(1^{d}\right)\right)=\sum_{j=0}^{d-1} 2^{j} \psi_{d}^{j}\left(\left(1^{d}\right)\right) .
$$

Note that by Theorem 3.1 (1),

$$
\sum_{j=0}^{d-1} \frac{\psi_{d}^{j}\left(\left(1^{d}\right)\right)}{q^{j}}=d ! \frac{T_{\left(1^{d}\right), 1}(q)}{q^{d}}=\frac{d !}{q^{d}}\left(\begin{array}{c}
q+d-1 \\
d
\end{array}\right)
$$

Evaluating (11) at $q=\frac{1}{2}$ implies

$$
\sum_{j=0}^{d-1} 2^{j} \psi_{d}^{j}\left(\left(1^{d}\right)\right)=2^{d} d !\left(\begin{array}{c}
d-\frac{1}{2} \\
d
\end{array}\right)=(2 d-1)(2 d-3) \cdots 3 \cdot 1=(2 d-1) ! !
$$

Therefore $\operatorname{dim} \bigoplus_{k=0}^{d} \Sigma_{d}^{k}=(2 d-1) ! !$.

(3) By definition we have

$$
\Sigma_{d}^{0} \cong \operatorname{sgn}_{d} \otimes \bigoplus_{j=0}^{d-1} H^{2 j}\left(\operatorname{PConf}_{d}\left(\mathbb{R}^{3}\right), \mathbb{Q}\right)
$$

In $[10$, Thm. 2.6] we showed that

$$
\bigoplus_{j=0}^{d-1} H^{2 j}\left(\operatorname{PConf}_{d}\left(\mathbb{R}^{3}\right), \mathbb{Q}\right) \cong \mathbb{Q}\left[S_{d}\right]
$$

where $\mathbb{Q}\left[S_{d}\right]$ is the regular representation. The claim follows from

$$
\operatorname{sgn}_{d} \otimes \mathbb{Q}\left[S_{d}\right] \cong \mathbb{Q}\left[S_{d}\right]
$$


Acknowledgements. The author thanks Weiyan Chen, Nir Gadish, Ofir Gorodetsky, Jeff Lagarias, Bob Lutz, John Stembridge, Phil Tosteson, Michael Zieve, and the referee for helpful conversations and suggestions on the manuscript.

\section{REFERENCES}

[1] A. Bodin, Number of irreducible polynomials in several variables over finite fields, Am. Math. Mon. 115 (2008), 653-660.

[2] L. Carlitz, The arithmetic of polynomials in a Galois field, Proc. Natl. Acad. Sci. U.S.A. 17 (1931), 120-122.

[3] _ The arithmetic of polynomials in a Galois field, Am. J. Math. 54 (1932), 39-50.

[4] , The distribution of irreducible polynomials in several indeterminates, Illinois J. Math. 7 (1963), 371-375.

[5] _ The distribution of irreducible polynomials in several indeterminates II, Canad. J. Math. 17 (1965), 261-266.

[6] T. Church, J. Ellenberg, and B. Farb, Representation stability in cohomology and asymptotics for families of varieties over finite fields, in Algebraic Topology: Applications and New Directions, Contemp. Math., vol. 620, American Mathematical Society, 2014, pp. 1-54.

[7] S. D. Cohen, The distribution of irreducible polynomials in several indeterminates over a finite field, P. Edinburgh Math. Soc. 16 (1968), 1-17.

[8] X.-D. Hou and G. Mullen, Number of irreducible polynomials and pairs of relatively prime polynomials in several variables over finite fields, Finite Fields Appl. 15 (2009), 304-331.

[9] T. Hyde, Cyclotomic factors of necklace polynomials, https://arxiv.org/abs/1811.08601, 2018.

[10] (2018).

[11] T. Hyde and J. C. Lagarias, Polynomial splitting measures and cohomology of the pure braid group, Arnold. Math. J. 3 (2017), 219-249.

[12] N. Metropolis and G.-C. Rota, Witt vectors and the algebra of necklaces, Adv. Math. 50 (1983), $95-125$.

[13] M. Rosen, Number theory in function fields, Graduate Texts in Mathematics, vol. 120, Springer Science \& Business Media, New York, 2013.

[14] N. J. A. Sloane, The On-Line Encyclopedia of Integer Sequences, 2018, https://oeis.org/ A088996, Accessed: 11-29-2018.

[15] R. P. Stanley, Combinatorial reciprocity theorems, Adv. Math. 14 (1974), 194-253.

[16] J. von zur Gathen, A. Viola, and K. Ziegler, Counting reducible, powerful, and relatively irreducible multivariate polynomials over finite fields, Siam J. Discrete Math. 27 (2013), 855-891.

[17] D. Wan, Zeta functions of algebraic cycles over finite fields, Manuscripta Math. 74 (1992), 413-444.

Trevor Hyde, University of Michigan, Dept. of mathematics, 530 Church St., Ann Arbor, MI 48109, USA

E-mail : tghyde@umich.edu 